\title{
Structural Control in Porous/Compact Multilayer Systems Grown by Magnetron Sputtering
}

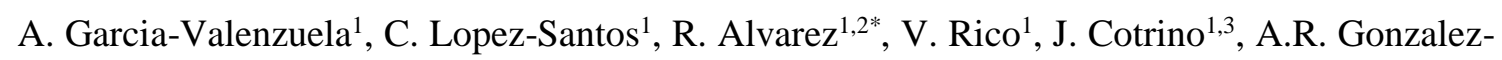
Elipe $^{1}$, A. Palmero ${ }^{1 *}$

${ }^{1}$ Instituto de Ciencia de Materiales de Sevilla (CSIC-US), Americo Vespucio, 49. 41092 Seville, Spain

${ }^{2}$ Departamento de Física Aplicada I. Universidad de Sevilla. Virgen de África, 7. 41011 Seville, Spain

${ }^{3}$ Departamento de Física Atómica, Molecular y Nuclear. Universidad de Sevilla. Avda. Reina Mercedes s/n. 41012 Seville, Spain

Abstract.-

In this work we analyze a phenomenon that takes place when growing magnetron sputtered porous/compact multilayer systems by alternating the oblique angle and the classical configuration geometries. We show that the compact layers develop numerous fissures rooted in the porous structures of the film below, in a phenomenon that amplifies when increasing the number of stacked layers. We demonstrate that these fissures emerge during growth due to the high roughness of the porous layers and the coarsening of a discontinuous interfacial region. To minimize this phenomenon, we have grown thin interlayers between porous and compact films under the impingement of energetic plasma ions, responsible for smoothing out the interfaces and inhibiting the formation of structural fissures. This method has been tested in practical situations for compact $\mathrm{TiO}_{2}$ /porous $\mathrm{SiO}_{2}$ multilayer systems, although it can be extrapolated to other materials and conditions.

Keywords: Oblique Angle Deposition; Magnetron Sputtering; Plasma-Assisted Deposition; Multilayers; $\mathrm{TiO}_{2} ; \mathrm{SiO}_{2}$

* Email: rafael.alvarez@icmse.csic.es; $\underline{\text { alberto.palmero@csic.es }}$ 


\section{Introduction}

Physical Vapor Deposition at oblique angles (OAD) is well known for promoting the growth of porous thin films with unique morphological features, e.g. high specific surface [1,2], tunable density $[3,4]$ or high pore connectivity $[5,6]$. These features make these films ideal candidates for applications in optical devices (due to their higher transparency [7]), plasmonics [8], electrical anisotropy [9], sensors [10,11], electrodes for solar cells [12] or biomedicine [13], among others. From a microstructural point of view, these films are formed by numerous well-separated tilted nanocolumnar structures that originate due to the oblique incidence of deposition species on a substrate, i.e., under conditions that enhance surface shadowing mechanisms during growth $[14,15]$. Even though the OAD geometry has been known since the beginning of the $20^{\text {th }}$ century [14], only recently has it been systematically explored by plasma-assisted magnetron sputtering (MS), widening its possibilities $[14,16,17,18]$. Relevant controllable quantities in this case are the tilt angle of the substrate with respect to the target [19] and the plasma gas pressure [20], both of them determining the momentum distribution and angle of incidence of the deposition species on the substrate. In fact, it is known that only at low pressures and for tilt angles of the substrate above $\sim 70^{\circ}$, a nanocolumnar microstructure may develop [19].

In all the aforementioned applications, the existence of a high void volume associated to the numerous pores open to the surface is essential for the good performance of OAD thin films. Yet, this type of porosity might introduce undesired collateral effects in multilayer systems, e.g., in photonic crystals or Bragg reflectors, where a strict periodicity between layers and sharp interfaces are required $[5,21]$. In this case, the large intercolumnar voids that characterize these films render a relatively high surface roughness [22], that might incidentally affect the growth of any layer on top and, hence, the morphology and nanostructure of the whole multilayer system. In the literature, most strategies devoted to smooth out rough surfaces rely either on a postprocessing treatment of the material $[23,24]$ or the impingement of energetic species, namely ions, during the growth of the film $[25,26,27]$. While the former is not recommended for the synthesis of multilayer stacks (it is much more efficient if the deposition takes place in one single 
run), the latter is well-known for promoting sputtering and mass transport processes that flatten the film surface [28]. Yet, ions may pose some drawbacks such as film densification and the decrease of the film porosity. For instance, in ref. [29], we analyzed the growth of $\mathrm{SiO}_{2}$ thin films by magnetron sputtering in the classical (non-oblique) configuration finding that, when the impingement rate of energetic negative oxygen ions was increased, the film morphology evolved from one characterized by sponge-like and vertically-aligned patterns to another characterized by an homogeneous and compact microstructure. Moreover, we reported a decrease in porosity of about one order of magnitude between these two situations, which would indeed represent a clear handicap in many applications relying on the porous nature of these films. To our knowledge, no in situ strategies have been yet specifically implemented to smooth out OAD thin films while maintaining most of its porosity, a procedure that would be of great relevance to achieve good structural control in porous multilayer structures.

In this paper, we report a remarkable and undesired phenomenon that takes place when piling up porous and compact films in multilayer stacks grown by MS. In this type of systems, we have found that compact layers develop numerous structural fissures rooted in the intercolumnar voids of the porous layers below. We also demonstrate that this phenomenon amplifies for increasing number of layers, leading to the loss of structural control over the whole system. In order to inhibit the formation of these fissures, we have developed a simple strategy that relies on the introduction of thin and homogeneous interlayers grown under the impingement of plasma ions between the porous and compact films. Although the results presented in this paper have been primarily tested for porous $\mathrm{SiO}_{2} /$ compact $\mathrm{TiO}_{2}$ multilayer systems at low temperatures, i.e., stacked structures that are typically employed to grow one dimensional photonic crystals, we also give some general insights to extrapolate our results to other cases and situations. 


\section{Experimental Setup}

Films were deposited in a cylindrical vacuum reactor (base pressure before deposition was below $5 \times 10^{-4} \mathrm{~Pa}$ ) equipped with two magnetron heads (Gencoa Ltd, Liverpool, UK) with silicon and titanium planar targets (diameter of $76.2 \mathrm{~mm}$ and $3 \mathrm{~mm}$ thick) as illustrated in Figure 1. Films were deposited onto $1 \times 1 \mathrm{~cm}^{2}$ planar (100) silicon substrates mounted on a rotatable holder $7 \mathrm{~cm}$ and $11.5 \mathrm{~cm}$ apart from the silicon and titanium targets, respectively. Same plasma conditions explored in ref. [29] were implemented: a pulsed unipolar power supply (Pinnacle Plus, Advanced Energy ${ }^{\circledR}$, USA) was employed, operated in a pulsed DC regime at a frequency of $80 \mathrm{KHz}$ and a duty cycle of $40 \%$, maintaining an electromagnetic power of $300 \mathrm{~W}$. A mixture of Ar (purity $99.995 \%$ ) and $\mathrm{O}_{2}$ (purity $99.995 \%$ ) was used for the depositions, with an argon partial pressure of $0.2 \mathrm{~Pa}$. The substrate tilt angle with respect to each target was set at $0^{\circ}$ when growing $\mathrm{TiO}_{2}$ compact layers and $85^{\circ}$ for the $\mathrm{SiO}_{2}$ porous films, respectively. Unless stated otherwise, the oxygen partial pressure to grow the $\mathrm{SiO}_{2}$ thin films was $0.02 \mathrm{~Pa}$, i.e. same conditions under which we demonstrated that ion impingement from the plasma was not influencing the nanostructural development of the films [29]. For some particular experiments, an energy-resolved mass spectrometer (EQP 500, Hiden Analytical Ltd) was also placed at the location of the films to assess the amount of ions from the plasma impinging on the film, as well as their energy. The spectrometer had an orifice with a diameter of $0.05 \mathrm{~mm}$, while the measurements were carried out in the energy range from 0 to $400 \mathrm{eV}$ for $\mathrm{TiO}_{2}$ and $\mathrm{SiO}_{2}$ films.

The surface roughness of the deposited layers was characterized with a Nanotec AFM microscope supplied with a Dulcinea electronics. The microscope was working in tapping mode by using high frequency levers. Au-covered SiN tips (Olympus) with a diameter of $40 \mathrm{~nm}$ were used. The resonance frequency of the tip was $20 \mathrm{kHz}$ and the spring constant $0.09 \mathrm{Nm}^{-1}$. The obtained surface maps were processed by means of the WSxM software [30]. Moreover, Field Emission Scanning Electron Microscopy (FESEM) pictures were recorded for each film using a Hitachi S4800 microsocope at the Instituto de Ciencia de Materiales de Sevilla (CSIC-US, Seville, Spain). 


\section{Results and Discussion}

\section{Microstructural propagation phenomenon}

Figure 2a (left image) depicts a cross-sectional SEM image of a compact $\mathrm{SiO}_{2} /$ compact $\mathrm{TiO}_{2}$ bilayer (monolayer thickness around $\sim 300 \mathrm{~nm}$ ), which was grown as a reference system. As expected, both layers show typical compact nanostructures and a rather sharp interface. Figure $2 b$ (left image) shows the SEM image of a porous $\mathrm{SiO}_{2} /$ compact $\mathrm{TiO}_{2}$ bilayer system with similar thickness. Remarkably, and even though the $\mathrm{TiO}_{2}$ thin films in both figures were grown under equivalent conditions, the reported images evidence clear morphological differences: while the bilayer structure in Figure 2a (left) is rather homogeneous, that in figure $2 \mathrm{~b}$ (left) depicts numerous structural fissures. This difference is more evident in figures $2 \mathrm{a}-\mathrm{b}$ (right images), which have been obtained after applying a border detection and contrast enhancement software to the images on the left. There, it is apparent the existence of structural fissures in the compact $\mathrm{TiO}_{2}$ film that root in the open porous structures of the $\mathrm{SiO}_{2}$ layer below. We dub this phenomenon microstructural propagation, an effect representing a serious shortcoming when piling up porous and compact layers in multistack structures. Indeed, a progressive loss of interface planarity and microstructural integrity are evidenced in the SEM micrograph in Figure 2c (left and right images) corresponding to a porous $\mathrm{SiO}_{2} /$ compact $\mathrm{TiO}_{2}$ eleven-layers multistack structure.

\section{Formation of an interfacial accommodation interlayer}

To understand the origin of the microstructural propagation phenomenon we analyze the correlation between surface features of the $\mathrm{SiO}_{2}$ porous film and the structural fissures in the $\mathrm{TiO}_{2}$ compact layer. In figure 3a-b we show the top SEM images of compact and porous $\mathrm{SiO}_{2}$ films, respectively. Unlike the highly smooth surface of the compact film in figure $3 \mathrm{a}$, the surface of the porous layer in figure $3 \mathrm{~b}$ developed numerous mounds associated to the tips of the nanocolumns. In fact, AFM topographic profiles of these films (shown as insets in the images) indicate that the porous layer possesses much higher height variations than the compact one, a result that suggests that the origin of the structural fissures in $\mathrm{TiO}_{2}$ is linked to the existence of large height variations 
on the surface it grows upon. To prove this connection, we have employed a well-known and tested growth model already presented in refs. [19, 31]. This model considers atomic shadowing as the main nanostructuration mechanism, in agreement with the Thornton's Structure Zone Model $[32,33]$ that describes the competition between shadowing processes and thermally activated migrations. In figures $4 \mathrm{a}$ and $4 \mathrm{~b}$ (left) we present the simulated compact $\mathrm{SiO}_{2} /$ compact $\mathrm{TiO}_{2}$ and porous $\mathrm{SiO}_{2} /$ compact $\mathrm{TiO}_{2}$ bilayer structures, which are in good agreement with the experimental images in figures $2 \mathrm{a}$ and $2 \mathrm{~b}$. Moreover, the series of snapshots presented in figure 4a (right) describing the first stages of growth of the compact $\mathrm{TiO}_{2}$ layer onto a compact $\mathrm{SiO}_{2}$ layer reveal the formation of a continuous interfacial accommodation layer that rapidly smooths out the underlying patterns and promotes a compact homogeneous growth. Similarly, the set of snapshots in figure $4 \mathrm{~b}$ (right) corresponding the growth of the compact $\mathrm{TiO}_{2}$ film onto the porous $\mathrm{SiO}_{2}$ layer shows that the deposition does not only occur on top of the nanocolumns but also inside the voids (pores), just below the average surface level. As a result of this partial conformal growth, the interfacial region presents clear discontinuities at the pore entrances. According to these simulations it appears that the coarsening of this discontinuous interfacial region generates numerous compact domains whose borders can be interpreted as structural fissures.

\section{Growth of a continuous accommodation interlayer on porous films}

Based on the analysis above, it seems that a requirement to preclude the microstructural propagation phenomenon is the growth of a continuous interfacial region to promote the development of a uniform $\mathrm{TiO}_{2}$ film on top. From an atomistic point of view, this cannot be accomplished whenever the film nanostructuration is controlled by surface shadowing processes: only by inducing atomic migration phenomena could the isolated domains formed at the entrances of the pores be connected. A first possibility to trigger these processes relies on thermally activated mechanisms (e.g., by heating up the sample during growth $[34,35])$. However, the Thornton's Structure Zone Model indicates that the condition $T_{f} / T_{m}>0.3$ must be fulfilled to ensure the dominance of surface diffusion, where $T_{f}$ is the film temperature during growth and $T_{m}$ 
the melting temperature of the deposited material. For $\mathrm{TiO}_{2}$, this condition implies $T_{f}>700 \mathrm{~K}$, a rather high value that would restrain its practical implementation for many applications.

An alternative to thermal activation in our conditions is the introduction of ion-induced mobility processes [25-27]. In fact, under MS conditions, ions from the magnetron plasma might play a critical role provided that they transfer enough energy to the growing film [36-38]. It has been mentioned in the introduction that under non-oblique (classical) conditions and at high oxygen fluxes the growth of $\mathrm{SiO}_{2}$ thin films by MS is strongly influenced by the impingement of energetic negative oxygen ions. These oxygen ions form at the silicon target surface and are accelerated towards the film with energies in the order of few hundred eVs, without applying any substrate bias [29]). Figure 5 shows the energy spectrum of negative oxygen ions measured by mass spectrometry when growing $\mathrm{SiO}_{2}$ films in the classical configuration with a high oxygen partial pressure $(0.25 \mathrm{~Pa}$, i.e. under conditions where negative ions were reported to effectively densify the film [29]). For comparison purposes, this figure includes the energy spectrum of the negative oxygen ions generated when growing a compact $\mathrm{TiO}_{2}$ thin film. In agreement with ref. [29], a high energy peak at around $300 \mathrm{eV}$ was found in the former case, whereas in the latter no trace of high energy negative oxygen ions was detected (the peaks at lower energies in the two cases correspond to ions at the plasma potential). A hypothesis of the present work is that a thin and compact $\mathrm{SiO}_{2}$ interlayer can be grown under the impingement of negative oxygen ions on top of the $\mathrm{SiO}_{2}$ porous surface, and that this interlayer promotes a continuous interfacial region when growing the compact $\mathrm{TiO}_{2}$ film. Keeping this purpose in mind, we have deposited several compact $\mathrm{SiO}_{2}$ layers with different thicknesses on top of a porous $\mathrm{SiO}_{2}$ layer. The cross-sectional images of these films in Figure 6a-e show no trace of structural fissures, suggesting that, effectively, ioninduced atomic mobility processes dominate over surface shadowing mechanisms, leading to the formation of a compact and continuous layer.

\section{Minimizing the microstructural propagation phenomenon among layers}

Once established the growth conditions of a continuous $\mathrm{SiO}_{2}$ layer, the next step is the determination of its minimum thickness to promote the growth of a homogeneous and compact 
$\mathrm{TiO}_{2}$ layer on top. For this purpose, we have studied the evolution of the surface roughness of this layer as a function of its thickness. According to the Dynamic Scaling Theory [39-41], the growth of a film can be described by a specific power law of quantities such as surface roughness, $r$, correlation length or grain size with the film thickness, $d$. Herein, we will characterize the growth by the relation $r \propto d^{\beta}$, where $\beta$ is the so-called growth exponent [42]. Figure 7 shows the evolution of surface roughness with thickness for a $\mathrm{SiO}_{2}$ porous layer (solid upper curve) deposited on a flat substrate. From these curves it is possible to derive $\beta \sim 0.8$. Figure 7 also shows the evolution of the surface roughness when a $\mathrm{SiO}_{2}$ compact layer is deposited on top of a porous film with thickness $\sim 280 \mathrm{~nm}$. It appears that when the compact layer thickness increases the surface roughness decreases until reaching a total thickness of $350 \mathrm{~nm}$, a point from which thickness increases again but now according to a different power law characterized by $\beta \sim 0.6$. Remarkably, and according to ref. [43], this latter value corresponds to a compact $\mathrm{SiO}_{2}$ growth, which implies that under negative oxygen ion bombardment an interfacial layer thickness of $\sim 25 \%$ of the porous layer below $(\sim 70 \mathrm{~nm} / 280 \mathrm{~nm})$ suffices to recover a compact growth mode. As a final step in this investigation, we analyze whether the strategy developed above is effective to minimize the microstructural propagation phenomenon. The cross-sectional SEM image in figure $8 \mathrm{a}$ (left) of a porous $\mathrm{SiO}_{2} /$ compact $\mathrm{TiO}_{2}$ bilayer with a continuous $\mathrm{SiO}_{2}$ interlayer clearly shows the absence of structural fissures. This is quite evident regarding the right column image, obtained after applying a border detection and contrast enhancement software. Finally, and as a final proof for the efficiency of the proposed strategy, in figure $8 \mathrm{~b}$ (left) we show a multilayer structure similar to that depicted in figure $2 \mathrm{c}$, but growing a $\sim 30 \mathrm{~nm} \mathrm{SiO}_{2}$ continuous interlayer on top of each $\sim 120 \mathrm{~nm}$ porous $\mathrm{SiO}_{2}$ structure. There, it is clear that the microstructure of all monolayers is similar and that the structural propagation phenomenon is inhibited, confirming the validity of the proposed strategy.

As a final remark, it is noteworthy that the proposed method relies on the growth of homogeneous and thin interlayers between porous and compact films by inducing atomic migration mechanisms. In our case, we have achieved this goal by promoting a relatively high energy ion impingement during the growth of a compact $\mathrm{SiO}_{2}$ interlayer. Yet, other approaches could also 
be possible to achieve the same scope, e.g. heating up the film substrate during growth or electrically biasing the film to attract positive ions. Although we have not attempted these other approaches, they are, in principle, valid and could be implemented depending on the characteristics of the multilayer system.

\section{Conclusions}

In this paper we have demonstrated the existence of an undesired effect, dubbed microstructural propagation phenomenon, which appears when porous layers grown at oblique angles are piled up with compact ones in multilayer systems. In these cases, the porous structure seems to promote the appearance of structural fissures throughout the compact films, in a process that becomes amplified when increasing the number of layers. Based on fundamental experiments and the results of a well-tested growth model, we have concluded that this phenomenon is caused by the existence of a discontinuous interfacial region that, upon coarsening, develops into numerous domains, whose borders can be appreciated as structural fissures in the compact layer. In order to minimize the propagation phenomenon, we propose depositing a continuous thin interfacial layer on top of each porous surface, previous to the deposition of the compact film. This strategy has been tested in a practical situation by depositing a periodic multilayer stack with porous $\mathrm{SiO}_{2} /$ compact $\mathrm{TiO}_{2}$ bilayer structure achieving well-defined periodic interfaces and a good replication of the morphology from the first to the last layer. These results demonstrate the validity of the developed approach to achieve good morphological control on the multilayer structure.

\section{Acknowledgements}

Authors acknowledge the University of Seville (VPPI-US), the Junta de Andalucía (TEP8067 and TEP5283) and the Ministry of Economy and Competitiveness of Spain (MAT2013-42900-P, MAT2013-40852-R, MAT2016-79866-R, MAT2015-69035-REDC) for financial support. 


\section{References}

[1] Borhani-Haghighi S, Khare C, Trocoli R, Dushina A, Kieschnick M, LaMantia F, Ludwig A 2016 Synthesis of nanostructured LiMn2O4 thin films by glancing angle deposition for Li-ion battery applications Nanotechnology 27455402.

[2] Khan S B, Hou M J, Shuang S, Zhang Z J 2017 Morphological influence of TiO2 nanostructures (nanozigzag, nanohelics and nanorod) on photocatalytic degradation of organic dyes Appl. Surf. Sci. 400 184-93.

[3] Alvarez R, Garcia-Martin J M, Garcia-Valenzuela A, Macias-Montero M, Ferrer F J, Santiso J, Rico V, Cotrino J, Gonzalez-Elipe A R and Palmero A 2016 Nanostructured Ti thin films by magnetron sputtering at oblique angles J. Phys. D: Appl. Phys. 49045303.

[4] Godinho V, Caballero-Hernandez J, Jamon D, Rojas T C, Schierholz R, Garcia-Lopez J, Ferrer F J, Fernandez A 2013 A new bottom-up methodology to produce silicon layers with a closed porosity nanostructure and reduced refractive index Nanotechnology 24275604.

[5] Oliva-Ramirez M, Gonzalez-Garcia L, Parra-Barranco J, Yubero F, Barranco A, GonzalezElipe A R 2013 Liquids Analysis with Optofluidic Bragg Microcavities ACS Appl. Mat. \& Interf. 5(14) 6743-50.

[6] Li S, Huang J, Cai L 2011 A porous silicon optical microcavity for sensitive bacteria detection Nanotechnology 22425502.

[7] Leem J W, Yu J S 2011 Glancing angle deposited ITO films for efficiency enhancement of a$\mathrm{Si}: \mathrm{H} / \mu \mathrm{c}-\mathrm{Si}: \mathrm{H}$ tandem thin film solar cells Optics Express 19(S3) A258-69.

[8] Vitrey A, Alvarez R, Palmero A, González M U, García-Martín J M 2017 Fabrication of Black-Gold Coatings by Glancing Angle Deposition With Sputtering Beilstein J. Nanotechnol. 8 434-39.

[9] Pedrosa P, Ferreira A, Cote J-M, Martin N, Arab M, Yazdi P, Lanceros-Mendez S, Vaz F 2017 Influence of the sputtering pressure on the morphological features and electrical resistivity anisotropy of nanostructured titanium films Appl. Surf. Sci. 420 681-90.

[10] Lee K, Shim Y S, Song Y G, Han S D, Lee Y S, Kang C Y 2017 Highly Sensitive Sensors Based on Metal-Oxide Nanocolumns for Fire Detection Sensors 17(2) 303. 
[11] Ollitrault J, Martin N, Rauch J Y, Sanchez J B, Berger F 2015 Improvement of ozone detection with GLAD WO3 films Mat. Lett. 155 1-3.

[12] Liu Y, Zhao Y, Feng Y, Shen J S, Liang X Y, Huang J, Min J H, Wang L J, Shi W M 2016 The influence of incident angle on physical properties of a novel back contact prepared by oblique angle deposition Appl. Surf. Sci. 363 252-8.

[13] Izquierdo-Barba I, García-Martín J M, Alvarez R, Palmero A, Esteban J, Pérez-Jorge C, Arcos D, Vallet-Regí M 2015 Nanocolumnar coatings with selective behavior towards osteoblast and Staphylococcus aureus proliferation Acta Biomater. 15 20-8.

[14] Barranco A, Borras A, Gonzalez-Elipe A R, Palmero A 2016 Perspectives on oblique angle deposition of thin films: From fundamentals to devices Prog. Mater. Sci. 76 59-153.

[15] Alvarez R, Lopez-Santos C, Parra-Barranco J, Rico V, Barranco A, Cotrino J, GonzalezElipe A R, Palmero A 2014 Nanocolumnar growth of thin films deposited at oblique angles: Beyond the tangent rule J. Vac. Sci. Technol. B 32(4) 041802.

[16] El Beainou R, Martin N, Potin V, Pedrosa P, Yazdi M A P, Billard A 2017 Correlation between structure and electrical resistivity of $\mathrm{W}-\mathrm{Cu}$ thin films prepared by GLAD co-sputtering Surf. Coat. Technol. 313 1-7.

[17] Sarkar S, Pradhan S K 2014 Tailoring of optical and wetting properties of sputter deposited silica thin films by glancing angle deposition Appl. Surf. Sci. 290 509-13.

[18] Dick B, Brett M J, Smy T 2003 Controlled growth of periodic pillars by glancing angle deposition J. Vac. Sci. Technol. B 21(1) 23.

[19] Alvarez R, Garcia-Martin J M, Macias-Montero M, Gonzalez-Garcia L, Gonzalez J C, Rico V, Perlich J, Cotrino J, González-Elipe A R, Palmero A 2013 Growth regimes of porous gold thin films deposited by magnetron sputtering at oblique incidence: from compact to columnar microstructures Nanotechnology 24045604.

[20] Palmero A, Rudolph H, Habraken F H P M 2006 Study of the gas rarefaction phenomenon in a magnetron sputtering system Thin Solid Films 515(2) 631-35.

[21] Galisteo-Lopez J F, Ibisate M, Sapienza R, Froufe-Perez L S, Blanco A, Lopez C 2011 Self-Assembled Photonic Structures Adv. Mat. 23(1), 30-69. 
[22] Potocnik J, Nenadovic M, Bundaleski N, Popovic M, Rakocevic Z 2016 Effect of thickness on optical properties of nickel vertical posts deposited by GLAD technique Optical Mat.

62 146-151.

[23] Chen X S, Xu Z Y, Wu K J, Zhang S N, Li H W, Meng Y C, Wang Z W, Li L Q, Ma X M 2016 Facile Peeling Method as a Post-Remedy Strategy for Producing an Ultrasmooth SelfAssembled Monolayer for High-Performance Organic Transistors Langmuir 32(37), 9492-500.

[24] Sauerbrey M et al. 2016 Ultrasmooth $\mathrm{Ru}(0001)$ Films as Templates for Ceria Nanoarchitectures Crystal Growth \& Design 16(8), 4216-24.

[25] Alvarez R, Palmero A, Prieto-López L O, Yubero F, Cotrino J, de la Cruz W, Rudolph H, Habraken F H P M, Gonzalez-Elipe A R 2010 Morphological evolution of pulsed laser deposited ZrO2ZrO2 thin films J. Appl. Phys. 107054311.

[26] Vogel N, Zieleniecki J, Koper I 2012 As flat as it gets: ultrasmooth surfaces from templatestripping procedures Nanoscale 4, 3820-32.

[27] Chen W, Chen K P, Thoreson M D, Kildishev A V, Shalaev V M 2010 Ultrathin, ultrasmooth, and low-loss silver films via wetting and annealing Appl. Phys. Lett. 97, 211107.

[28] Mayr S G, Moske M, Samwer K, Taylor M E, Atwater H A 1999 The role of particle energy and pulsed particle flux in physical vapor deposition and pulsed-laser deposition Appl. Phys. Lett. 75, 4091-3.

[29] Macias-Montero M, Garcia-Garcia F J, Alvarez R, Gil-Rostra J, Gonzalez J C, Cotrino J, Gonzalez-Elipe A R, Palmero A 2012 Influence of plasma-generated negative oxygen ion impingement on magnetron sputtered amorphous $\mathrm{SiO} 2$ thin films during growth at low temperatures J. Appl. Phys. 111, 054312.

[30] Horcas I, Fernandez R, Gomez-Rodriguez J M, Colchero J, Gomez-Herrero J, Baro A M 2007 WSxM: a software for scanning probe microscopy and a tool for nanotechnology Rev. Sci. Instrum. 78013705.

[31] Alvarez R, Romero-Gomez P, Gil-Rostra J, Cotrino J, Yubero F, Palmero A, GonzalezElipe A R 2010 On the microstructure of thin films grown by an isotropically directed deposition flux J. Appl. Phys. 108(6) 064316.

[32] Thornton J A 1974 Influence of apparatus geometry and deposition conditions on the structure and topography of thick sputtered coatings J. Vac. Sci. Technol. 11666. 
[33] Thornton J A 1975 Influence of substrate temperature and deposition rate on structure of thick sputtered Cu coatings J. Vac. Sci. Technol. 12830.

[34] Deniz D, Lad R J 2011 Temperature threshold for nanorod structuring of metal and oxide films grown by glancing angle deposition J. Vac. Sci. Technol. A 29(1) 011020.

[35] Nandi R, Major S S 2017 The mechanism of growth of ZnO nanorods by reactive sputtering Appl. Surf. Sci. 399, 305-12.

[36] Lin Y W, Huang J H, Yu G P, Hsiao C N, Chen F Z 2015 Influence of ion bombardment on structure and properties of TiZrN thin film Appl. Surf. Sci. 354 155-60.

[37] Welzel T, Ellmer K 2013 Comparison of ion energies and fluxes at the substrate during magnetron sputtering of $\mathrm{ZnO}: \mathrm{Al}$ for dc and $\mathrm{rf}$ discharges J. Phys. D: Appl. Phys. 46(31), 315202 .

[38] Ellmer K, Welzel T 2012 Reactive magnetron sputtering of transparent conductive oxide thin films: Role of energetic particle (ion) bombardment J. Mat. Res. 27(5), 765-79.

[39] Pelliccione M and Lu T-M 2008 Evolution of Thin Film Morphology: Modeling and Simulations Springer Series: Materials Science, vol. 108, Springer, Berlin.

[40] Barabàsi A L, Stanley H E 1995 Fractal Concepts in Surface Growth Cambridge University Press, Cambridge, England.

[41] Oliveira T J, Reis F D A A 2007 Effects of grains' features in surface roughness scaling $J$. Appl. Phys. 101(6) 063507.

[42] Karabacak T 2011 Thin-film growth dynamics with shadowing and re-emission effects $J$. Nanophotonics 5, 052501.

[43] Elsholz F, Schöll E, Scharfenorth C, Seewald G, Eichler H J, Rosenfeld A 2005 Roughness evolution in thin-film growth of $\mathrm{SiO} 2$ and Nb2O5 J. Appl. Phys. 98, 103516. 


\section{Figure Caption}

Figure 1: Experimental Setup.

Figure 2: Left) Cross-section SEM micrographs of: a) compact $\mathrm{SiO}_{2}(\sim 330 \mathrm{~nm}$ thick)/ compact $\mathrm{TiO}_{2}\left(\sim 300 \mathrm{~nm}\right.$ thick) bilayer, b) porous $\mathrm{SiO}_{2}(\sim 220 \mathrm{~nm}$ thick $) /$ compact $\mathrm{TiO}_{2}(\sim 235 \mathrm{~nm}$ thick $)$ bilayer, c) multilayer structure formed by the stacking of porous $\mathrm{SiO}_{2}(\sim 60 \mathrm{~nm}$ thick $) /$ compact $\mathrm{TiO}_{2}$ ( $\sim 60 \mathrm{~nm}$ thick) layers. Right) Same images after a treatment with a border detection and contrast enhancement software.

Figure 3: a) Top SEM image of a compact $\mathrm{SiO}_{2}$ thin film. b) Top SEM image of a porous $\mathrm{SiO}_{2}$ thin film. Linear topographic profiles as measured by AFM are shown as insets (in case b, the profile corresponds to the particle flux direction). Roughness of both surfaces is included.

Figure 4: Simulation of a) the $\mathrm{SiO}_{2}$ compact $/ \mathrm{TiO}_{2}$ compact bilayer and b) the porous $\mathrm{SiO}_{2} /$ compact $\mathrm{TiO}_{2}$ bilayer. Right images correspond to snapshots of the first stages of growth of the $\mathrm{TiO}_{2}$ thin film in either case.

Figure 5: Energy spectra of the negative oxygen ions when depositing $\mathrm{SiO}_{2}$ and $\mathrm{TiO}_{2}$ in our conditions, as obtained by an energy-resolved mass spectrometer.

Figure 6: Cross-sectional SEM images of porous $\mathrm{SiO}_{2}$ layers with similar thickness along with different compact $\mathrm{SiO}_{2}$ layers on top grown under the impingement of plasma ions.

Figure 7: Surface roughness evolution of porous $\mathrm{SiO}_{2}$ layers as a function of thickness. The influence of growing a compact $\mathrm{SiO}_{2}$ layer under the impingement of plasma ions is also depicted. The transition from a pure columnar to a compact growth mode is evidenced.

Figure 8: Left) Cross-section $\mathrm{SEM}$ micrographs of: a) porous $\mathrm{SiO}_{2} /$ compact $\mathrm{SiO}_{2}$ interlayer/compact $\mathrm{TiO}_{2}$, b) multilayer structure formed by the stacking of porous $\mathrm{SiO}_{2} /$ compact interlayer $\mathrm{SiO}_{2} /$ compact $\mathrm{TiO}_{2}$ layers. Right) Same images after a treatment with a border detection and contrast enhancement software. 
Figure 1

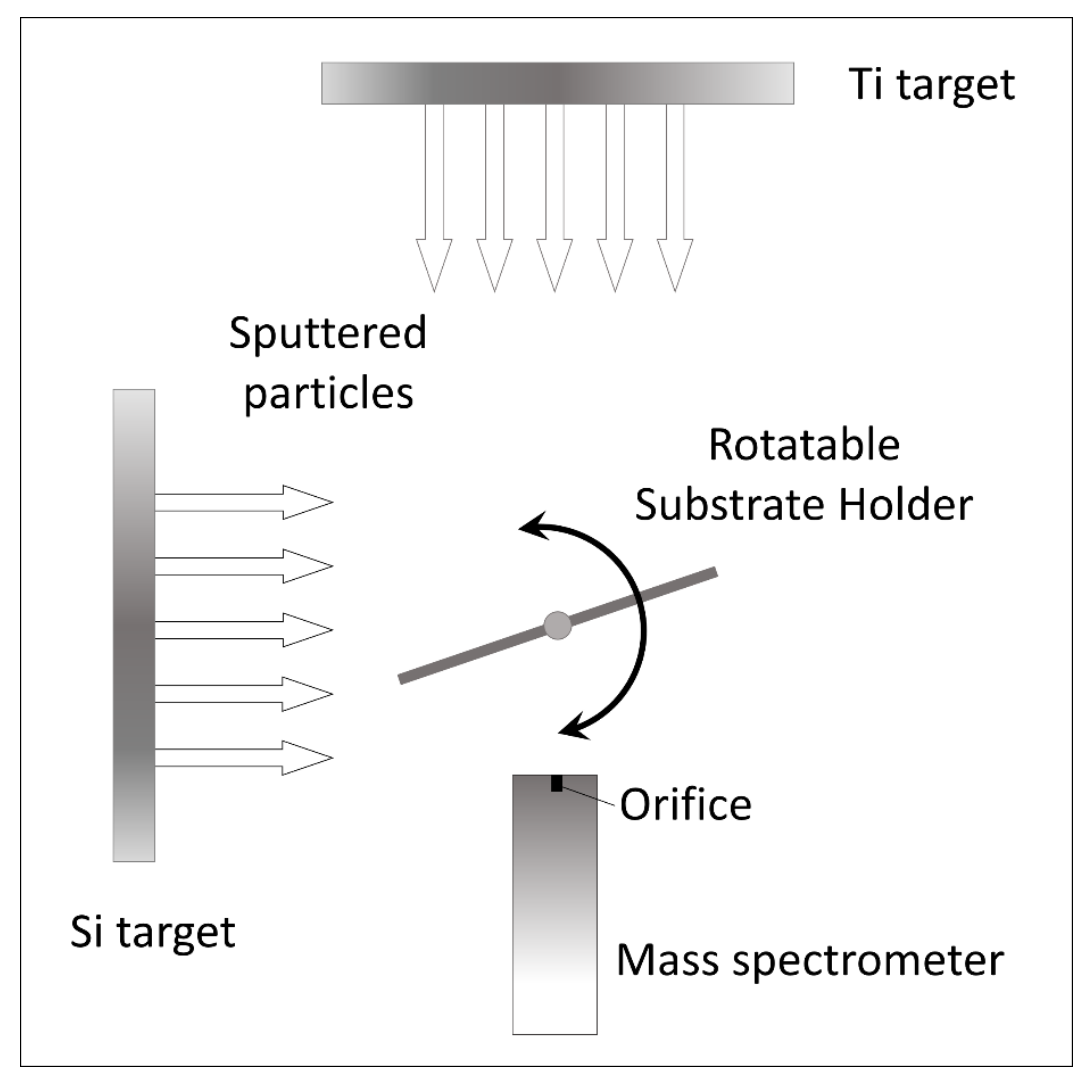


Figure 2
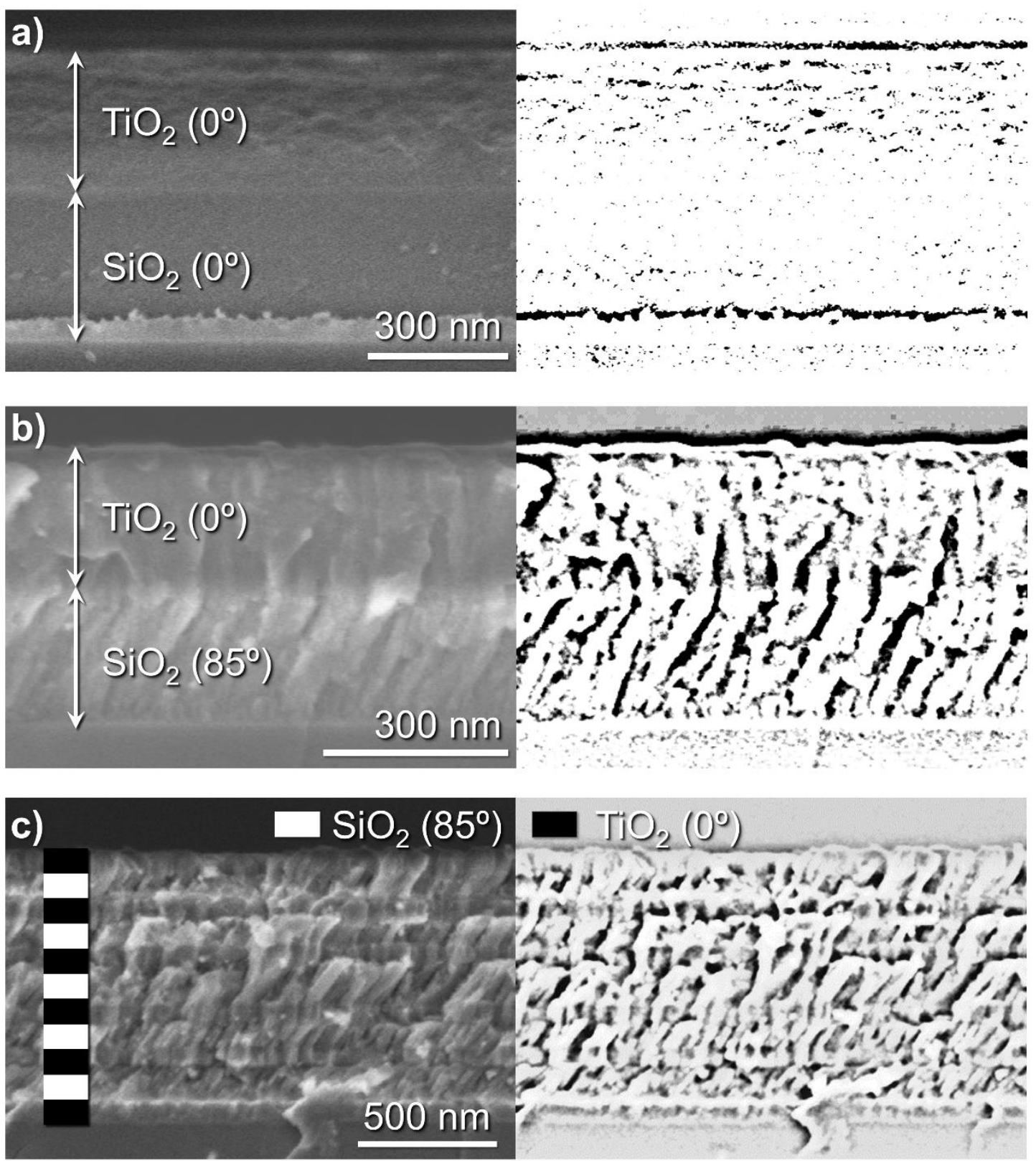
Figure 3
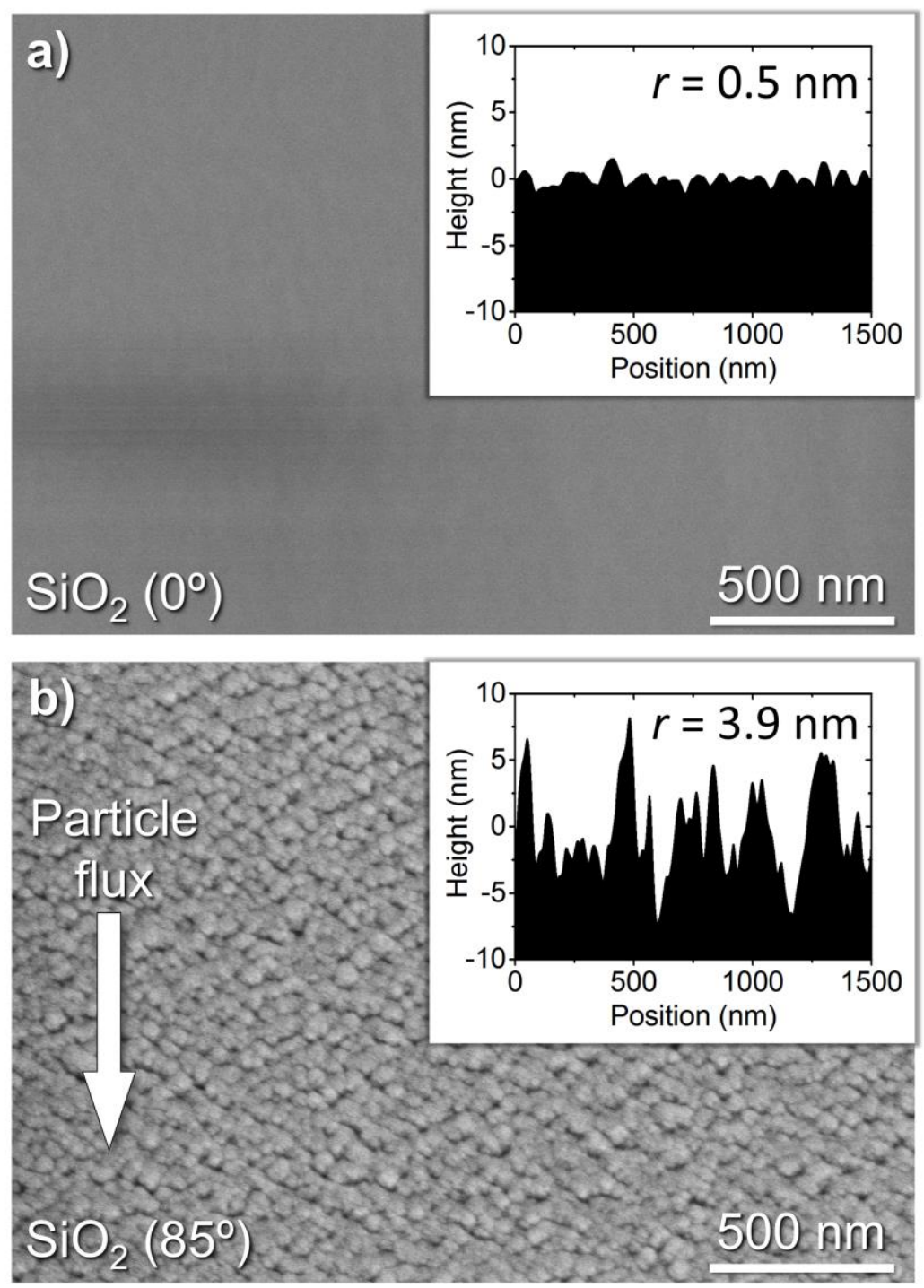
Figure 4
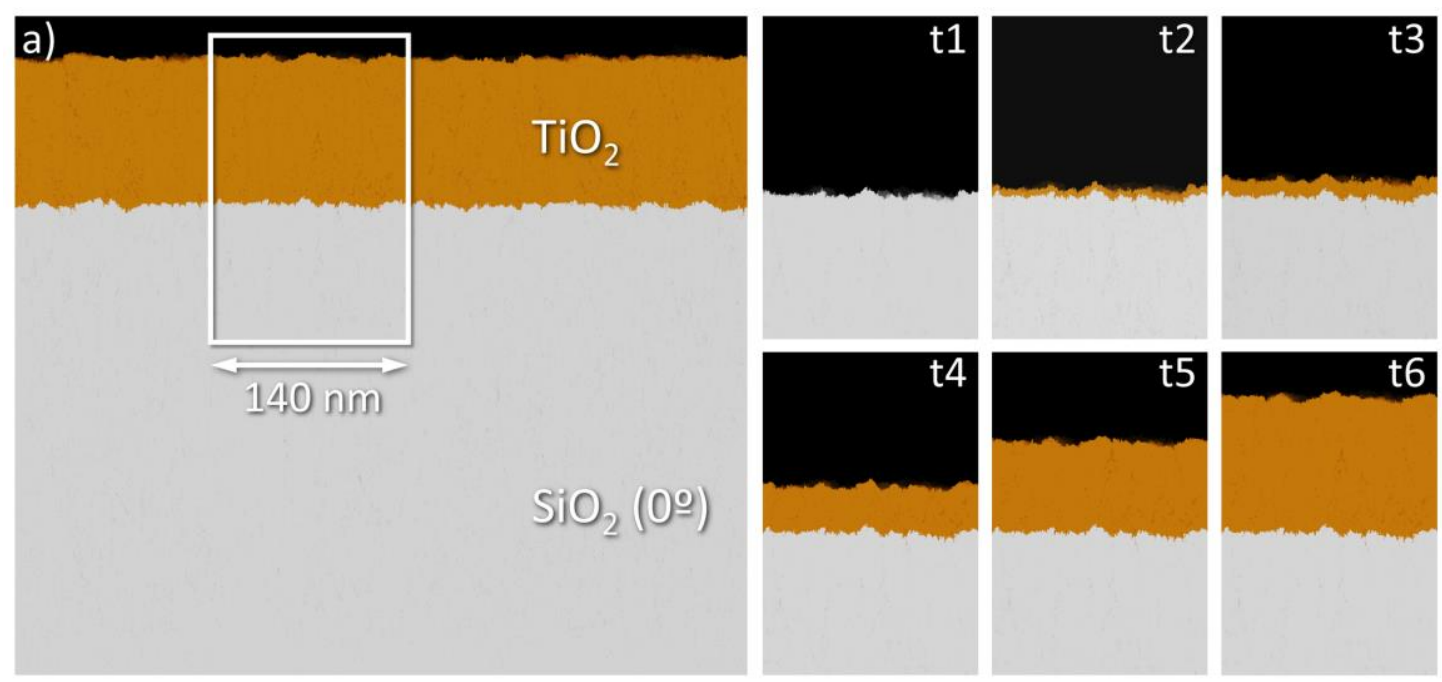

b).
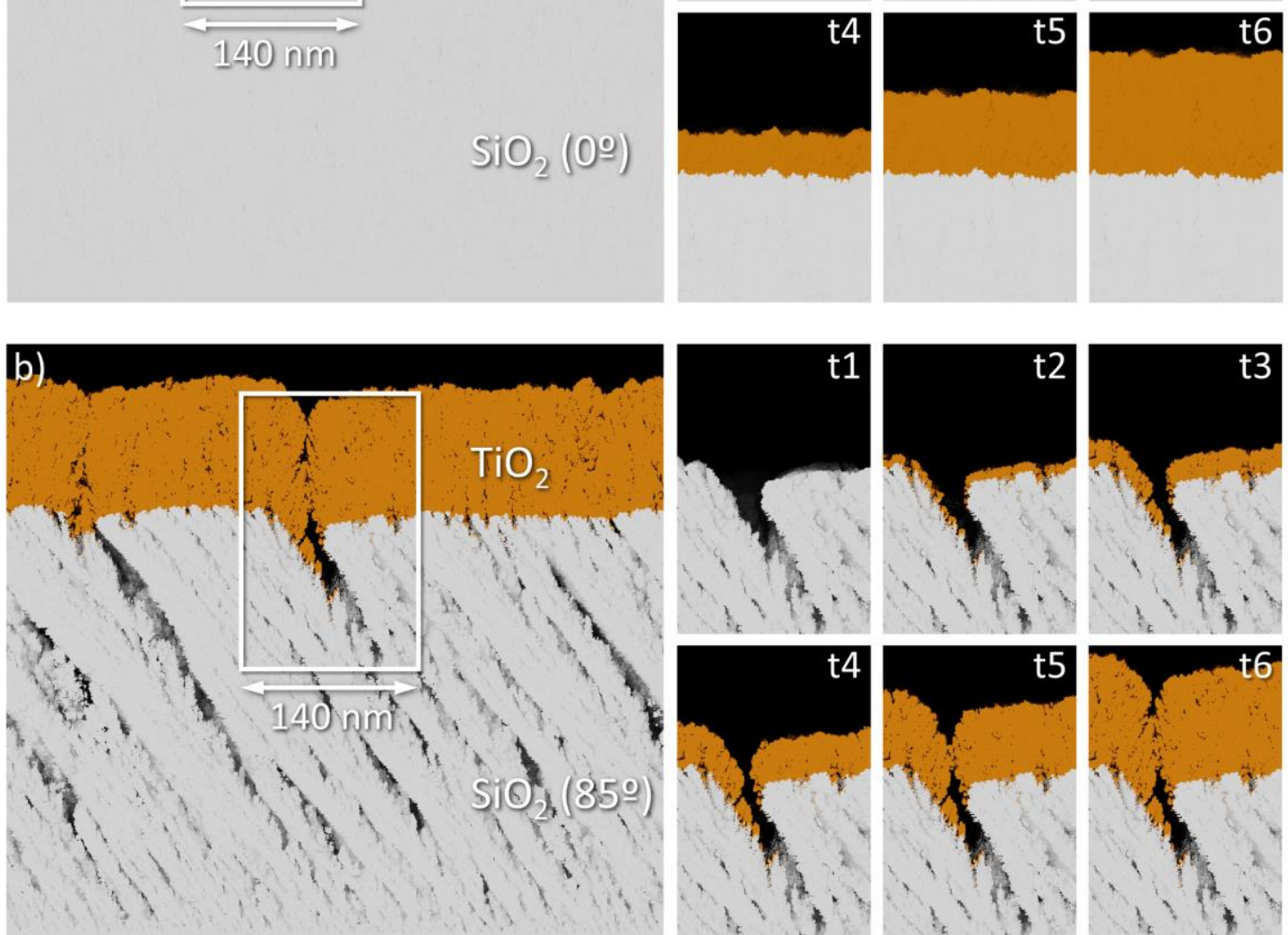
Figure 5

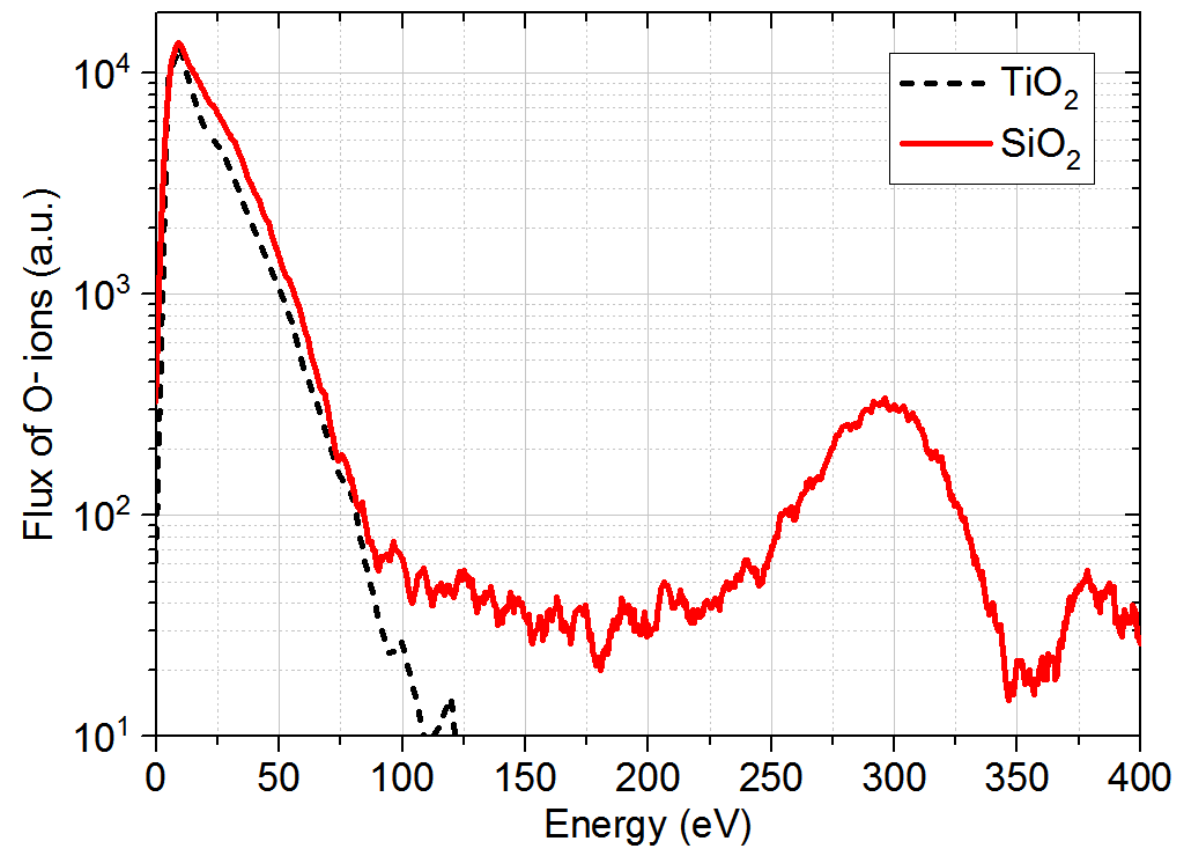


Figure 6
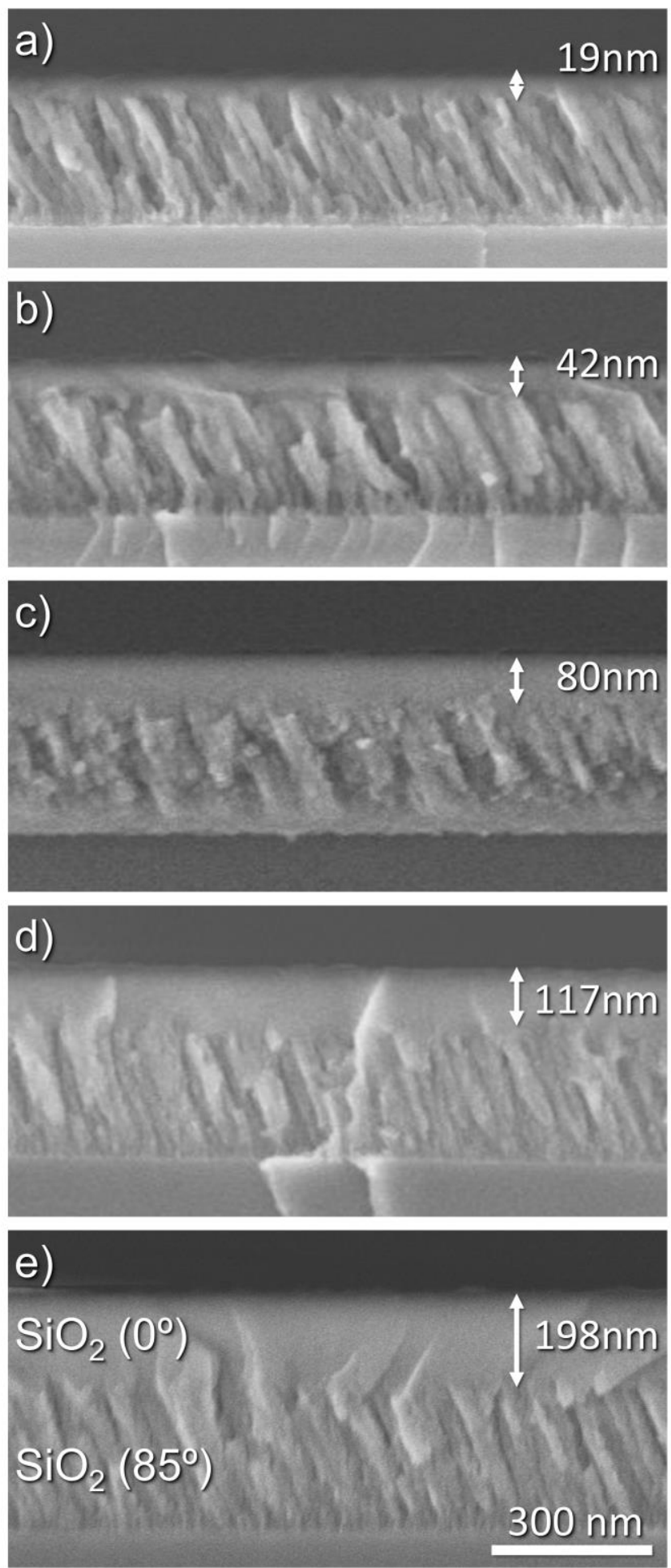
Figure 7

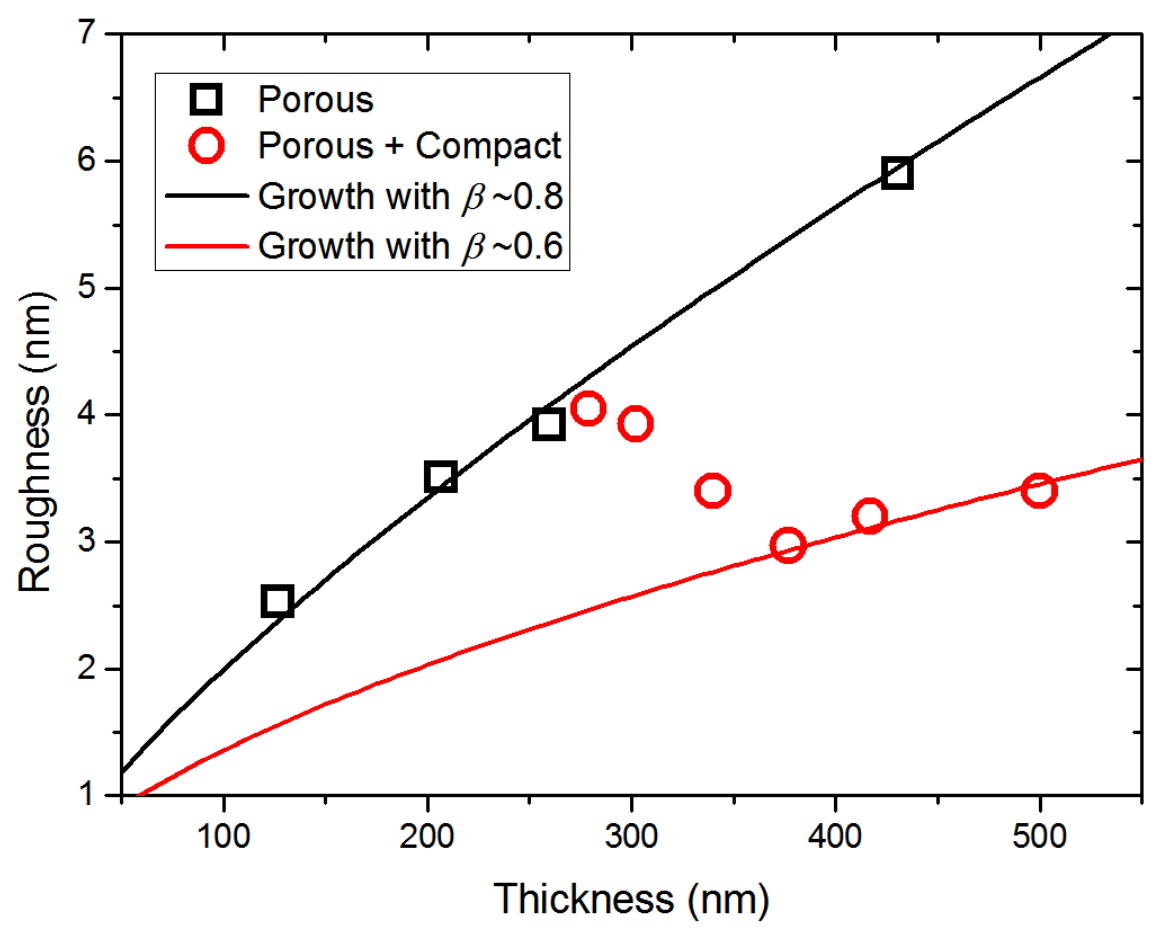


Figure 8
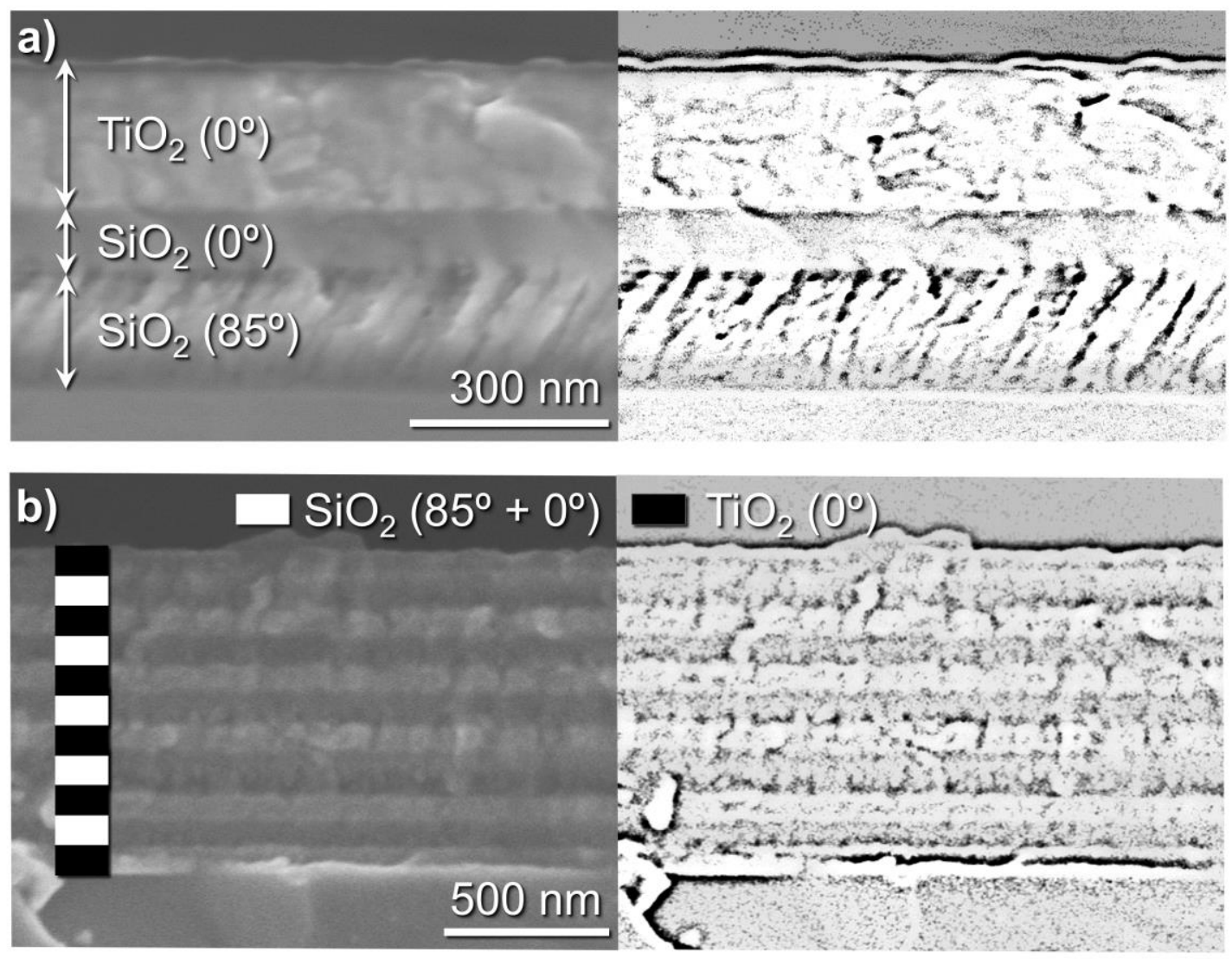